\title{
Attracting and invariant sets of nonlinear neutral differential equations with delays
}

Shujun Long*

${ }^{\text {*Correspondence: }}$
longer207@yahoo.com.cn
College of Mathematics and
Information Science, Leshan Normal
University, Leshan, 614004,
P.R. China

\begin{abstract}
In this paper, we study the attracting and invariant sets for a class of nonlinear neutral differential equations with delays. By using the properties of $\mathcal{M}$-matrix, a new delay differential-difference inequality is established. Based on the new inequality, we get the global attracting and invariant sets and the sufficient condition ensuring the exponential stability in Lyapunov sense of nonlinear neutral differential equations with delays. Our results are independent of time delays and do not require the differentiability, boundedness of the derivative of delay functions and the boundedness of activation functions. Two examples are presented to illustrate the effectiveness of our conclusion.
\end{abstract}

Keywords: attracting set; invariant set; stability; neutral; differential-difference inequality; delays

\section{Introduction}

Delay effects exist widely in many real-world models such as the SEIRS epidemic model [1] and neural networks [2-5]. The existence of time delays may destroy a stable system and cause sustained oscillations, bifurcation or chaos and thus could be harmful. Therefore, it is of prime importance to consider the effect of delays on the dynamical behaviors of the system. Recently, there are many authors who consider the effect of delays on the stability in Lyapunov sense of the system with time delays [2-11]. In addition, another type of time delays, namely neutral-type time delays, has recently drawn much attention in research [12-21]. In fact, many practical delay systems can be modeled as differential systems of neutral type whose differential expression includes not only the derivative term of the current state but also the derivative of the past state, such as partial element equivalent circuits and transmission lines in electrical engineering, controlled constrained manipulators in mechanical engineering, neural networks models, and population dynamics (see [22] and references therein).

The works [12-22] mentioned above are focused on studying the stability in Lyapunov sense of the neutral differential equations, which requires the existence and uniqueness of equilibrium points. However, in many real physical systems, especially in nonlinear and non-autonomous dynamical systems, the equilibrium point sometimes does not exist. Therefore, an interesting subject is to discuss the stability in Lagrange sense. Basically, the goal of the study on global stability in Lagrange sense is to determine global attracting sets. Once a global attracting set is found, a rough bound of periodic states and chaotic attractors can be estimated. For this reason, some significant works have been done on the 
techniques and methods of determining the invariant set and attracting set for various differential systems [23-30]. In these works mentioned before, there is only one paper [25] that considers a positive invariant set and a global attracting set for nonlinear neutral differential systems with delays, but the boundedness of activation functions is required.

It is well known that differential inequalities are very important tools for investigating the dynamical behavior of differential equations (see [11, 20, 21, 26, 28, 31-34]). Xu et al. developed a delay differential inequality with the impulsive initial conditions and derived some sufficient conditions to determine the invariant set and the global attracting set for a class of nonlinear non-autonomous functional differential systems with impulsive effects [28]. In [32], Eduardo Liz et al. developed a generalized Halanay inequality and derived some sufficient conditions for the existence and stability of almost periodic solutions for quasilinear delay systems. In [20], Xu et al. developed the singular impulsive delay differential inequality and transformed the $n$-dimensional impulsive neutral differential equation to a $2 n$-dimensional singular impulsive delay differential equation and derived some sufficient conditions ensuring the global exponential stability in Lyapunov sense of a nonlinear impulsive neutral differential equation with time-varying delays, but they assumed that the discontinuous points of the derivative of the solution belonged to the first kind. As we all know, the discontinuous points of the derivative of continuous functions may not be the first kind. In addition, we know that LMI method is another effective tool for investigating the dynamical behavior of a differential system [14, 15, 35]. The results given in the LMI form are dependent on time delays, so we must give additional constraint conditions such as differentiability or boundedness of the derivative of delay functions on the time-varying delays. However, the conditions given in the form of $\mathcal{M}$-matrix are usually independent of the time delays, thus, the time delays are harmless. Motivated by the before discussions, our objective in this paper is to improve the inequality established in [28] and [32] so that it is effective for neutral differential equation. By establishing a new delay differential-difference inequality, without assuming that the discontinuous points of the derivative of the solution belong to the first kind, the global attracting and invariant sets and the sufficient condition ensuring the global exponential stability in Lyapunov sense of a nonlinear neutral differential equations with delays are obtained. Our results are independent of the time delays, and do not require the differentiability, boundedness of the derivative of delay functions and the boundedness of activation functions. Two examples are presented to illustrate the effectiveness of our conclusion.

\section{Model description and preliminaries}

Throughout this paper, we use the following notations. Let $R_{+}^{n}$ be the space of $n$ dimensional nonnegative real column vectors, $R^{n}$ be the space of $n$-dimensional real column vectors, $\mathcal{N} \triangleq\{1,2, \ldots, n\}$, and $R^{m \times n}$ denote the set of $m \times n$ real matrices. Usually $E$ denotes an $n \times n$ unit matrix. For $A, B \in R^{m \times n}$, the notation $A \geq B(A>B)$ means that each pair of corresponding elements of $\mathrm{A}$ and $\mathrm{B}$ satisfies the inequality ' $\geq(>)$.' Especially, $A \in R^{m \times n}$ is called a nonnegative matrix if $A \geq 0$, and $z$ is called a positive vector if $z>0$. $A_{r}$ denotes the $r$ th row vector of the matrix $A$.

$C[X, Y]$ denotes the space of continuous mappings from the topological space $X$ to the topological space $Y$. Especially, $C \triangleq C\left[[-\tau, 0], R^{n}\right]$ denotes the family of all continuous $R^{n}$ valued functions, where $\tau>0$.

$P C\left[, R^{n}\right]=\left\{\varphi: J \rightarrow R^{n}\right.$ is continuous for all but at most a finite number of points $t \in$ $J$, and at these points $t \in J, \varphi\left(t^{+}\right)$and $\varphi\left(t^{-}\right)$exist, $\left.\varphi\left(t^{+}\right)=\varphi(t)\right\}$, where $J \subset R$ is a bounded 
interval, $\varphi\left(t^{+}\right)$and $\varphi\left(t^{-}\right)$denote the right-hand and left-hand limits of the function $\varphi(t)$, respectively. Especially, let $P C \triangleq P C\left[[-\tau, 0], R^{n}\right]$.

For $A \in R^{n \times n}, x \in R^{n}, \phi \in C$ and $\varphi$ is a continuous function on $\left[t_{0}-\tau,+\infty\right)$, we define

$$
\begin{aligned}
& |A|=\left(\left|a_{i j}\right|\right)_{n \times n}, \quad[x]^{+}=\left(\left|x_{1}\right|, \ldots,\left|x_{n}\right|\right)^{T}, \\
& {[\phi]_{\tau}^{+}=\left[[\phi]^{+}\right]_{\tau}, \quad\left[\phi_{i}\right]_{\tau}=\sup _{-\tau \leq s \leq 0}\left\{\phi_{i}\left(t_{0}+s\right)\right\},} \\
& {[\varphi(t)]_{\tau}=\left(\left[\varphi_{1}(t)\right]_{\tau}, \ldots,\left[\varphi_{n}(t)\right]_{\tau}\right)^{T}, \quad[\varphi(t)]_{\tau}^{+}=\left[[\varphi(t)]^{+}\right]_{\tau},} \\
& {\left[\varphi_{i}(t)\right]_{\tau}=\sup _{-\tau \leq s \leq 0}\left\{\varphi_{i}(t+s)\right\}, \quad t \geq t_{0}, i \in \mathcal{N},}
\end{aligned}
$$

and $D^{+} \varphi(t)$ denotes the upper-right-hand derivative of $\varphi(t)$ at time $t$.

For $\varphi \in C$, we introduce the following norm:

$$
\|\varphi\|_{\tau}=\max _{1 \leq i \leq n}\left\{\max _{-\tau \leq s \leq 0}\left|\varphi_{i}(s)\right|\right\} .
$$

In this paper, we consider the following nonlinear neutral differential equation with time-varying delays:

$$
\left\{\begin{aligned}
&\left(x_{i}(t)-\sum_{j=1}^{n} c_{i j} x_{j}\left(t-r_{i j}(t)\right)\right)^{\prime}=-d_{i} x_{i}(t)+\sum_{j=1}^{n} a_{i j} f_{j}\left(x_{j}(t)\right) \\
&+\sum_{j=1}^{n} b_{i j} g_{j}\left(x_{j}\left(t-\tau_{i j}(t)\right)\right)+J_{i}, \quad t \geq t_{0}, \\
& x_{i}\left(t_{0}+s\right)=\phi_{i}(s), \quad-\tau \leq s \leq 0, i \in \mathcal{N},
\end{aligned}\right.
$$

where $\tau, a_{i j}, b_{i j}, c_{i j}, d_{i}$ and $J_{i}$ are constants, $\tau_{i j}(t), r_{i j}(t), f_{j}(t), g_{j}(t) \in C[R, R], i, j \in \mathcal{N}, r_{i j}(t)$ is differentiable, and $\tau_{i j}(t), r_{i j}(t)$ satisfy

$$
0 \leq \tau_{i j}(t) \leq \tau, \quad 0<r_{i j}(t) \leq \tau
$$

the initial function $\phi(s)=\left(\phi_{1}(s), \ldots, \phi_{n}(s)\right)^{T} \in C$.

Throughout this paper, the solution $x(t)$ of $(1)$ with the initial condition $\phi \in C$ is denoted by $x\left(t, t_{0}, \phi\right)$ or $x_{t}\left(t_{0}, \phi\right)$, where $x_{t}\left(t_{0}, \phi\right)=x\left(t+s, t_{0}, \phi\right), s \in[-\tau, 0]$.

Definition 1 The set $S \subset C$ is called a positive invariant set of (1) if, for any initial value $\phi \in S$, we have the solution $x_{t}\left(t_{0}, \phi\right) \in S$ for $t \geq t_{0}$.

Definition 2 The set $S \subset C$ is called a global attracting set of (1) if, for any initial value $\phi \in C$, the solution $x_{t}\left(t_{0}, \phi\right)$ converges to $S$ as $t \rightarrow+\infty$. That is,

$$
\operatorname{dist}\left(x_{t}\left(t_{0}, \phi\right), S\right) \rightarrow 0 \quad \text { as } t \rightarrow+\infty
$$

where $\operatorname{dist}(\varphi, S)=\inf _{\psi \in S} \operatorname{dist}(\varphi, \psi), \operatorname{dist}(\varphi, \psi)=\sup _{s \in[-\tau, 0]}|\varphi(s)-\psi(s)|$, for $\varphi \in C$.

Definition 3 The zero solution of (1) is said to be globally exponentially stable in Lyapunov sense if there exist constants $\lambda>0$ and $M \geq 1$ such that for any solution $x\left(t, t_{0}, \phi\right)$ with the initial condition $\phi \in C$,

$$
\left\|x_{t}\left(t_{0}, \phi\right)\right\|_{\tau} \leq M\|\phi\|_{\tau} e^{-\lambda\left(t-t_{0}\right)}, \quad t \geq t_{0} .
$$


Definition 4 ([36]) Let the matrix $D=\left(d_{i j}\right)_{n \times n}$ have non-positive off-diagonal elements (i.e., $d_{i j} \leq 0, i \neq j$ ), then each of the following conditions is equivalent to the statement ' $D$ is a nonsingular $\mathcal{M}$-matrix'.

(i) All the leading principle minors of $D$ are positive.

(ii) $D=C-M$ and $\rho\left(C^{-1} M\right)<1$, where $M \geq 0, C=\operatorname{diag}\left\{c_{1}, \ldots, c_{n}\right\}$.

(iii) The diagonal elements of $D$ are all positive and there exists a positive vector $d$ such that $D d>0$ or $D^{T} d>0$.

For a nonsingular $\mathcal{M}$-matrix $D$, we denote $\Omega_{M}(D) \triangleq\left\{z \in R^{n} \mid D z>0, z>0\right\}$.

For a nonnegative matrix $A \in R^{n \times n}$, let $\rho(A)$ be the spectral radius of $A$. Then $\rho(A)$ is an eigenvalue of $A$ and its eigenspace is denoted by

$$
\Omega_{\rho}(A) \triangleq\left\{z \in R^{n} \mid A z=\rho(A) z\right\},
$$

which includes all positive eigenvectors of $A$ provided that the nonnegative matrix $A$ has at least one positive eigenvector (see Ref. [37]).

Lemma 1 ([36]) If $A \geq 0$ and $\rho(A)<1$, then

(a) $(E-A)^{-1} \geq 0$;

(b) there is a positive vector $z \in \Omega_{\rho}(A)$ such that $(E-A) z>0$.

\section{Main results}

Based on Lemma 1 in [28] and Theorem 2.1 in [32], we develop the following delay differential-difference inequality with the $P C$-value initial condition such that it is effective for neutral differential equation with delays.

Theorem 1 Let $\sigma<b \leq+\infty$, and $u \in C\left[[\sigma, b), R_{+}^{n}\right], 4 \omega \in C\left[[\sigma, b), R_{+}^{p}\right]$ satisfy

$$
\left\{\begin{array}{l}
D^{+} u(t) \leq P u(t)+Q[u(t)]_{\tau}+G \omega(t)+H[\omega(t)]_{\tau}+\eta, \\
\omega(t) \leq M u(t)+N[u(t)]_{\tau}+R[\omega(t)]_{\tau}+I, \quad t \in[\sigma, b), \\
u(t)=\phi(t), \quad \omega(t)=\varphi(t), \quad t \in[\sigma-\tau, \sigma],
\end{array}\right.
$$

where $\phi \in P C\left[[\sigma-\tau, \sigma], R_{+}^{n}\right], \varphi \in P C\left[[\sigma-\tau, \sigma], R_{+}^{p}\right], P=\left(p_{i j}\right)_{n \times n}, p_{i j} \geq 0$, for $i \neq j, Q=$ $\left(q_{i j}\right)_{n \times n} \geq 0, G=\left(g_{i j}\right)_{n \times p} \geq 0, H=\left(h_{i j}\right)_{n \times p} \geq 0, M=\left(m_{i j}\right)_{p \times n} \geq 0, N=\left(n_{i j}\right)_{p \times n} \geq 0, R=$ $\left(r_{i j}\right)_{p \times p} \geq 0, \eta=\left(\eta_{1}, \ldots, \eta_{n}\right)^{T} \geq 0$ and $I=\left(I_{1}, \ldots, I_{p}\right)^{T} \geq 0$. Suppose that $\rho(R)<1$ and $\Pi=-\left(P+Q+(G+H)(E-R)^{-1}(M+N)\right)$ is an $\mathcal{M}$-matrix, then the solution of (4) has the following property:

$$
\left\{\begin{array}{l}
u(t) \leq k z e^{-\lambda(t-\sigma)}+\hat{\eta}, \\
\omega(t) \leq k \tilde{z} e^{-\lambda(t-\sigma)}+\hat{I}, \quad t \in[\sigma, b),
\end{array}\right.
$$

provided that the initial conditions satisfy

$$
\left\{\begin{array}{l}
u(t) \leq k z e^{-\lambda(t-\sigma)}+\hat{\eta}, \\
\omega(t) \leq k \tilde{z} e^{-\lambda(t-\sigma)}+\hat{I}, \quad t \in[\sigma-\tau, \sigma],
\end{array}\right.
$$


where

$$
\begin{aligned}
& k \geq 0, \quad z \in \Omega_{M}(\Pi), \quad \tilde{z} \triangleq\left(E-R e^{\lambda \tau}\right)^{-1}\left(M+N e^{\lambda \tau}\right) z, \\
& \hat{\eta} \triangleq \Pi^{-1} \eta+\Pi^{-1}(G+H)(E-R)^{-1} I, \\
& \hat{I} \triangleq(E-R)^{-1}(M+N) \Pi^{-1} \eta+\left((E-R)^{-1}(M+N) \Pi^{-1}(G+H)(E-R)^{-1}+(E-R)^{-1}\right) I,
\end{aligned}
$$

and the positive constant $\lambda$ is determined by the following inequalities:

$$
\rho\left(e^{\lambda \tau} R\right)<1 \quad \text { and } \quad\left(\lambda E+P+Q e^{\lambda \tau}+\left(G+H e^{\lambda \tau}\right)\left(E-R e^{\lambda \tau}\right)^{-1}\left(M+N e^{\lambda \tau}\right)\right) z<0 .
$$

Proof Since $\Pi$ is an $\mathcal{M}$-matrix, there exists a vector $z \in \Omega_{M}(\Pi)$ such that $\Pi z>0$, that is $\left(P+Q+(G+H)(E-R)^{-1}(M+N)\right) z<0$. By using continuity and combining with $\rho(R)<1$, we know there exists a positive constant $\lambda$ satisfying (7).

We at first shall prove that for any positive $\varepsilon$

$$
\left\{\begin{array}{l}
u(t)<(k+\varepsilon) z e^{-\lambda(t-\sigma)}+\hat{\eta} \triangleq \xi(t), \\
\omega(t)<(k+\varepsilon) \tilde{z} e^{-\lambda(t-\sigma)}+\hat{I} \triangleq \zeta(t), \quad t \in[\sigma, b) .
\end{array}\right.
$$

If inequality (8) is not true, from (6) and $u \in C\left[[\sigma, b), R_{+}^{n}\right], \omega \in C\left[[\sigma, b), R_{+}^{p}\right]$, then there must be a constant $t^{*}>\sigma$ and some integer $m, r$ such that

$$
\begin{aligned}
& u_{m}\left(t^{*}\right)=\xi_{m}\left(t^{*}\right), \quad D^{+} u_{m}\left(t^{*}\right) \geq \xi^{\prime}\left(t^{*}\right), \\
& u_{i}(t) \leq \xi_{i}(t), \quad t \in\left[\sigma-\tau, t^{*}\right], i=1, \ldots, n
\end{aligned}
$$

or

$$
\omega_{r}\left(t^{*}\right)=\zeta_{r}\left(t^{*}\right), \quad \omega_{j}(t) \leq \zeta_{j}(t), \quad t \in\left[\sigma-\tau, t^{*}\right], j=1, \ldots, p
$$

By using (4), (7), (9) and (10), we have

$$
\begin{aligned}
D^{+} u_{m}\left(t^{*}\right) \leq & P_{m} u\left(t^{*}\right)+Q_{m}\left[u\left(t^{*}\right)\right]_{\tau}+G_{m} \omega\left(t^{*}\right)+H_{m}\left[\omega\left(t^{\prime \prime}\right)\right]_{\tau}+\eta_{m} \\
\leq & P_{m}\left[(k+\varepsilon) z e^{-\lambda\left(t^{*}-\sigma\right)}+\hat{\eta}\right]+Q_{m}\left[(k+\varepsilon) z e^{\lambda \tau} e^{-\lambda\left(t^{*}-\sigma\right)}+\hat{\eta}\right] \\
& +G_{m}\left[(k+\varepsilon) \tilde{z} e^{-\lambda\left(t^{*}-\sigma\right)}+\hat{I}\right]+H_{m}\left[(k+\varepsilon) \tilde{z} e^{\lambda \tau} e^{-\lambda\left(t^{*}-\sigma\right)}+\hat{I}\right]+\eta_{m} \\
= & (k+\varepsilon)\left[P+Q e^{\lambda \tau}+\left(G+H e^{\lambda \tau}\right)\left(E-R e^{\lambda \tau}\right)^{-1}\left(M+N e^{\lambda \tau}\right) z\right]_{m} e^{-\lambda\left(t^{*}-\sigma\right)} \\
& +\left[\left(P+Q+(G+H)(E-R)^{-1}(M+N)\right) \Pi^{-1} \eta\right]_{m}+\eta_{m} \\
& \left.+\left[(P+Q) \Pi^{-1}(G+H)(E-R)^{-1} I\right]_{m}+\left[(G+H)(E-R)^{-1}\right) I\right]_{m} \\
& +\left[(G+H)(E-R)^{-1}(M+N) \Pi^{-1}(G+H)(E-R)^{-1} I\right]_{m} \\
< & \left.-\lambda(k+\varepsilon) z_{m} e^{-\lambda\left(t^{*}-\sigma\right)}+\left[-\Pi \Pi^{-1} \eta\right]_{m}+\eta_{m}+\left[(G+H)(E-R)^{-1}\right) I\right]_{m} \\
& +\left[\left(-\Pi-(G+H)(E-R)^{-1}(M+N)\right) \Pi^{-1}(G+H)(E-R)^{-1} I\right]_{m} \\
& +\left[(G+H)(E-R)^{-1}(M+N) \Pi^{-1}(G+H)(E-R)^{-1} I\right]_{m} \\
= & -\lambda(k+\varepsilon) z_{m} e^{-\lambda\left(t^{*}-\sigma\right)}=\xi^{\prime}\left(t^{*}\right) .
\end{aligned}
$$


This contradicts the second inequality in (9), so the first inequality in (8) holds. Therefore, we have to assume that (10) holds and we shall obtain another contradiction. Next, we consider three cases.

Case 1. The elements of the $M_{r}$ and $N_{r}$ are not all zero. Without loss of generality, we let $m_{r l}>0,1 \leq l \leq n$. Then, by using (4), (10) and the first inequality in (8), we have

$$
\begin{aligned}
\omega_{r}\left(t^{*}\right) \leq & M_{r} u\left(t^{*}\right)+N_{r}\left[u\left(t^{*}\right)\right]_{\tau}+R_{r}\left[\omega\left(t^{*}\right)\right]_{\tau}+I_{r} \\
= & m_{r l} u_{l}\left(t^{*}\right)+\sum_{j \neq l} m_{r j} u_{j}\left(t^{*}\right)+N_{r}\left[u\left(t^{*}\right)\right]_{\tau}+R_{r}\left[\omega\left(t^{*}\right)\right]_{\tau}+I_{r} \\
< & m_{r l} \xi_{l}\left(t^{*}\right)+\sum_{j \neq l} m_{r j} \xi_{j}\left(t^{*}\right)+N_{r}\left[u\left(t^{*}\right)\right]_{\tau}+R_{r}\left[\omega\left(t^{*}\right)\right]_{\tau}+I_{r} \\
\leq & M_{r}\left[(k+\varepsilon) z e^{-\lambda\left(t^{*}-\sigma\right)}+\hat{\eta}\right]+N_{r}\left[(k+\varepsilon) z e^{\lambda \tau} e^{-\lambda\left(t^{*}-\sigma\right)}+\hat{\eta}\right] \\
& +R_{r}\left[(k+\varepsilon) \tilde{z} e^{\lambda \tau} e^{-\lambda\left(t^{*}-\sigma\right)}+\hat{I}\right]+I_{r} \\
= & (k+\varepsilon)\left[\left(M+N e^{\lambda \tau}+R e^{\lambda \tau}\left(E-R e^{\lambda \tau}\right)^{-1}\left(M+N e^{\lambda \tau}\right)\right) z\right]_{r} e^{-\lambda\left(t^{*}-\sigma\right)} \\
& +\left[\left(M+N+R(E-R)^{-1}(M+N)\right) \Pi^{-1} \eta\right]_{r}+\left[R(E-R)^{-1} I\right]_{r}+I_{r} \\
& +\left[\left((M+N) \Pi^{-1}(G+H)(E-R)^{-1}\right.\right. \\
& \left.\left.+R(E-R)^{-1}(M+N) \Pi^{-1}(G+H)(E-R)^{-1}\right) I\right]_{r} \\
= & (k+\varepsilon)\left[\left(E-R e^{\lambda \tau}\right)^{-1}\left(M+N e^{\lambda \tau}\right) z\right]_{r} e^{-\lambda\left(t^{*}-\sigma\right)} \\
& +\left[(E-R)^{-1}(M+N) \Pi^{-1} \eta\right]_{r} \\
& +\left[\left((E-R)^{-1}(M+N) \Pi^{-1}(G+H)(E-R)^{-1}+(E-R)^{-1}\right) I\right]_{r} \\
= & (k+\varepsilon) \tilde{z}_{r} e^{-\lambda\left(t^{*}-\sigma\right)}+\hat{I}_{r}=\zeta_{r}\left(t^{*}\right) .
\end{aligned}
$$

Which contradicts the first equality in (10), so under this case, the second inequality in (8) holds.

Case 2. The elements of the $M_{r}$ and $N_{r}$ are all zero, but the elements of the $R_{r}$ are not all zero. Without loss of generality, we let $r_{r h}>0,1 \leq h \leq p$. Combining with $\omega \in C\left[[\sigma, b), R_{+}^{p}\right]$ and the monotonicity of $\zeta(t)$, from (10) and $[\omega(t)]_{\tau}=\sup _{-\tau \leq s \leq 0} \omega(t+s)$, we know there must exist $t^{*}-\tau \leq t_{1}, \ldots, t_{p} \leq t^{*}$ such that

$$
\left[\omega\left(t^{*}\right)\right]_{\tau}=\sup _{t^{*}-\tau \leq t \leq t^{*}} \omega(t)=\left(\omega_{1}\left(t_{1}\right), \ldots, \omega_{p}\left(t_{p}\right)\right)^{T}<\left(\zeta_{1}\left(t^{*}-\tau\right), \ldots, \zeta_{p}\left(t^{*}-\tau\right)\right)^{T}
$$

By using (4) and (13), we have

$$
\begin{aligned}
\omega_{r}\left(t^{*}\right) & \leq R_{r}\left[\omega\left(t^{*}\right)\right]_{\tau}+I_{r} \\
& =r_{r h}\left[\omega_{h}\left(t^{*}\right)\right]_{\tau}+\sum_{j \neq h} r_{r j}\left[\omega_{j}\left(t^{*}\right)\right]_{\tau}+I_{r} \\
& <r_{r h} \zeta_{h}\left(t^{*}-\tau\right)+\sum_{j \neq h} r_{r j} \zeta_{j}\left(t^{*}-\tau\right)+I_{r} \\
& =R_{r} \zeta\left(t^{*}-\tau\right)+I_{r} \\
& =R_{r}\left[(k+\varepsilon) \tilde{z} e^{\lambda \tau} e^{-\lambda\left(t^{*}-\sigma\right)}+\hat{I}\right]+I_{r}
\end{aligned}
$$




$$
\begin{aligned}
= & (k+\varepsilon)\left[R e^{\lambda \tau}\left(E-R e^{\lambda \tau}\right)^{-1}\left(M+N e^{\lambda \tau}\right) z\right]_{r} e^{-\lambda\left(t^{*}-\sigma\right)} \\
& +\left[R(E-R)^{-1}(M+N) \Pi^{-1} \eta\right]_{r} \\
& +\left[R(E-R)^{-1}(M+N) \Pi^{-1}(G+H)(E-R)^{-1} I\right]_{r}+\left[R(E-R)^{-1} I\right]_{r}+I_{r} \\
= & (k+\varepsilon)\left[\left(E-R e^{\lambda \tau}\right)^{-1}\left(M+N e^{\lambda \tau}\right) z\right]_{r} e^{-\lambda\left(t^{*}-\sigma\right)}+\left[(E-R)^{-1}(M+N) \Pi^{-1} \eta\right]_{r} \\
& +\left[(E-R)^{-1}(M+N) \Pi^{-1}(G+H)(E-R)^{-1} I\right]_{r}+\left[(E-R)^{-1} I\right]_{r} \\
& -(k+\varepsilon)\left[\left(E-R e^{\lambda \tau}\right)\left(E-R e^{\lambda \tau}\right)^{-1}\left(M+N e^{\lambda \tau}\right) z\right]_{r} e^{-\lambda\left(t^{*}-\sigma\right)} \\
& -\left[(E-R)(E-R)^{-1}(M+N) \Pi^{-1} \eta\right]_{r} \\
& -\left[(E-R)(E-R)^{-1}(M+N) \Pi^{-1}(G+H)(E-R)^{-1} I\right]_{r} \\
= & (k+\varepsilon) \tilde{z}_{r} e^{-\lambda\left(t^{*}-\sigma\right)}+\hat{I}_{r}=\zeta_{r}\left(t^{*}\right),
\end{aligned}
$$

which contradicts the first equality in (10); so under this case, the second inequality in (8) holds.

Case 3. The elements of the $M_{r}, N_{r}$ and $R_{r}$ are all zero, then the conclusion of the second inequality in (5) is trivial.

From the above analysis, we know (8) is true for all $t \in[\sigma, b)$. Letting $\varepsilon \rightarrow 0$ in (8), we can get (5).

The proof is complete.

Remark 1 Suppose that $M=N=0, R=0, I=0$ in Theorem 1, then we get Lemma 1 in [28]. Suppose that $J=0, I=0$ in Theorem 1, then we get Theorem 2.1 in [32].

For the model (1), we introduce the following assumptions:

$\left(A_{1}\right)$ The functions $f_{j}(\cdot), g_{j}(\cdot)$ are Lipschitz continuous, i.e., there are positive constants $k_{j}, l_{j}, j \in \mathcal{N}$ such that for all $s_{1}, s_{2} \in R$

$$
\left|f_{j}\left(s_{1}\right)-f_{j}\left(s_{2}\right)\right| \leq k_{j}\left|s_{1}-s_{2}\right|, \quad\left|g_{j}\left(s_{1}\right)-g_{j}\left(s_{2}\right)\right| \leq l_{j}\left|s_{1}-s_{2}\right| .
$$

$\left(A_{2}\right)$ Let $\|C\|<1$ and $\hat{\Pi}=-\left(-2 D+(D+\hat{A}+\hat{B})(E-|C|)^{-1}\right)$ be a nonsingular $\mathcal{M}$ matrix, where $D=\operatorname{diag}\left\{d_{1}, \ldots, d_{n}\right\}>0, \hat{A}=\left(\left|a_{i j}\right| k_{j}\right)_{n \times n}, \hat{B}=\left(\left|b_{i j}\right| l_{j}\right)_{n \times n}$. Let $\hat{J}=|A|[f(0)]^{+}+$ $|B|[g(0)]^{+}+[J]^{+}$.

Theorem 2 Assume that $\left(A_{1}\right),\left(A_{2}\right)$ hold. Then $S=\left\{\phi \in C \mid[\phi]_{\tau}^{+} \leq(E-|C|)^{-1} \hat{\Pi}^{-1} \hat{J}\right\}$ is a global attracting set of (1).

Proof Under the conditions $\left(A_{1}\right),\left(A_{2}\right)$, from $[7,8]$, we know the solution $x\left(t, t_{0}, \phi\right)$ of $(1)$ exists globally. We denote

$$
\begin{aligned}
u(t) & = \begin{cases}{[x(t)-C x(t-r(t))]^{+},} & t \geq t_{0}, \\
W\left[(E-|C|)[\phi]_{\tau}^{+}\right]^{+}, & t_{0}-\tau \leq t \leq t_{0},\end{cases} \\
\omega(t) & =[x(t)]^{+}, \quad t \geq t_{0}-\tau,
\end{aligned}
$$

where $W=\operatorname{diag}\left\{w_{1}, \ldots, w_{n}\right\} \geq 0$ such that $\left[\phi\left(t_{0}\right)-C \phi\left(-r\left(t_{0}\right)\right)\right]^{+}=W\left[(E-|C|)[\phi]_{\tau}^{+}\right]^{+}$. 
Then, for $t \geq t_{0}$, from (1) and $\left(A_{1}\right)$, we calculate the upper-right-hand derivative $D^{+} u(t)$ along the solutions of (1),

$$
\begin{aligned}
D^{+} u_{i}(t)= & \operatorname{sgn}\left(x_{i}(t)-\sum_{j=1}^{n} c_{i j} x_{j}\left(t-r_{i j}(t)\right)\right)\left\{-d_{i}\left(x_{i}(t)-\sum_{j=1}^{n} c_{i j} x_{j}\left(t-r_{i j}(t)\right)\right)\right. \\
& -\sum_{j=1}^{n} d_{i} c_{i j} x_{j}\left(t-r_{i j}(t)\right)+\sum_{j=1}^{n} a_{i j}\left[f_{j}\left(x_{j}(t)\right)-f_{j}(0)\right] \\
& \left.+\sum_{j=1}^{n} b_{i j}\left[g_{j}\left(x_{j}\left(t-\tau_{i j}(t)\right)\right)-g_{j}(0)\right]+\sum_{j=1}^{n}\left(a_{i j} f_{j}(0)+b_{i j} g_{j}(0)\right)+J_{i}\right\} \\
\leq & -d_{i}\left|x_{i}(t)-\sum_{j=1}^{n} c_{i j} x_{j}\left(t-r_{i j}(t)\right)\right|+\sum_{j=1}^{n}\left|a_{i j}\right| k_{j}\left|x_{j}(t)\right|+\sum_{j=1}^{n}\left|b_{i j}\right| l_{j}\left|x_{j}\left(t-\tau_{i j}(t)\right)\right| \\
& +\sum_{j=1}^{n} d_{i}\left|c_{i j}\right|\left|x_{j}\left(t-r_{i j}(t)\right)\right|+\sum_{j=1}^{n}\left(\left|a_{i j}\right|\left|f_{j}(0)\right|+\left|b_{i j}\right|\left|g_{j}(0)\right|\right)+\left|J_{i}\right| \\
\leq & -d_{i} u_{i}(t)+\sum_{j=1}^{n}\left|a_{i j}\right| k_{j} \omega_{j}(t)+\sum_{j=1}^{n}\left(\left|b_{i j}\right| l_{j}+d_{i}\left|c_{i j}\right|\right)\left[\omega_{j}(t)\right]_{\tau} \\
& +\sum_{j=1}^{n}\left(\left|a_{i j}\right|\left|f_{j}(0)\right|+\left|b_{i j}\right|\left|g_{j}(0)\right|\right)+\left|J_{i}\right|, \quad i \in \mathcal{N}, t \geq t_{0} .
\end{aligned}
$$

So, from (16) and $\left(A_{2}\right)$, we get

$$
D^{+} u(t) \leq-D u(t)+\hat{A} \omega(t)+(\hat{B}+D|C|)[\omega(t)]_{\tau}^{+}+\hat{J}, \quad t \geq t_{0} .
$$

On the other hand, we have

$$
\begin{aligned}
\omega_{i}(t) & =\left|x_{i}(t)\right|=\left|\left(x_{i}(t)-\sum_{j=1}^{n} c_{i j} x_{j}\left(t-r_{i j}(t)\right)\right)+\sum_{j=1}^{n} c_{i j} x_{j}\left(t-r_{i j}(t)\right)\right| \\
& \leq\left|\left(x_{i}(t)-\sum_{j=1}^{n} c_{i j} x_{j}\left(t-r_{i j}(t)\right)\right)\right|+\sum_{j=1}^{n}\left|c_{i j}\right|\left|x_{j}\left(t-r_{i j}(t)\right)\right| \\
& \leq u_{i}(t)+\sum_{j=1}^{n}\left|c_{i j}\right|\left[\omega_{j}(t)\right]_{\tau}, \quad t \geq t_{0} .
\end{aligned}
$$

That is,

$$
\omega(t) \leq u(t)+|C|[\omega(t)]_{\tau}^{+}, \quad t \geq t_{0}
$$

From $\left(A_{2}\right)$, Definition 4 and Lemma 1 , we have $(E-|C|)^{-1} \geq 0, \hat{\Pi}^{-1} \geq 0$, and so

$$
\varrho \triangleq \hat{\Pi}^{-1} \hat{J} \geq 0, \quad v \triangleq(E-|C|)^{-1} \hat{\Pi}^{-1} \hat{J} \geq 0 .
$$

Furthermore, for $z \in \Omega_{M}(\hat{\Pi})$, we have

$$
\left(-2 D+(D+\hat{A}+\hat{B})(E-|C|)^{-1}\right) z<0 .
$$


By using continuity, we can find a positive constant $\lambda$ such that

$$
\begin{aligned}
& \rho\left(e^{\lambda \tau}|C|\right)<1 \text { and } \\
& \left(\lambda E-2 D+\left(D+\hat{A}+\hat{B} e^{\lambda \tau}\right)\left(E-|C| e^{\lambda \tau}\right)^{-1}\right) z<0 \quad \text { for } z \in \Omega_{M}(\hat{\Pi})
\end{aligned}
$$

and we know

$$
\tilde{z} \triangleq\left(E-|C| e^{\lambda \tau}\right)^{-1} z>0 .
$$

From (15) and the initial conditions in (1): $x\left(t_{0}+s\right)=\phi(s), s \in[-\tau, 0]$, where $\phi \in C$, we can get

$$
u(t) \leq k_{0} z, \quad \omega(t) \leq k_{0} \tilde{z}, \quad k_{0}=\frac{\max _{1 \leq i \leq n}\left\{w_{i} \sum_{j=1}^{n} \varsigma_{i j}\|\phi\|_{\tau}\right\}}{\min _{1 \leq i \leq n, 1 \leq j \leq n}\left\{z_{i}, \tilde{z}_{j}\right\}}, t_{0}-\tau \leq t \leq t_{0},
$$

where $\left(\varsigma_{i j}\right)_{n \times n}=|(E-|C|)|$. From (20), (22), we know

$$
u(t) \leq k_{0} z e^{-\lambda\left(t-t_{0}\right)}+\varrho, \omega(t) \leq k_{0} \tilde{z} e^{-\lambda\left(t-t_{0}\right)}+v, \quad t_{0}-\tau \leq t \leq t_{0} .
$$

From (17), (19), (23), $\left(A_{2}\right)$ and Theorem 1 , we get

$$
u(t) \leq k_{0} z e^{-\lambda\left(t-t_{0}\right)}+\varrho, \quad \omega(t) \leq k_{0} \tilde{z} e^{-\lambda\left(t-t_{0}\right)}+v, \quad t \geq t_{0}
$$

From (24), we know the conclusion is true. The proof is complete.

If $J=0, f(0)=g(0)=0$ in the model (1), then we know the model (1) has an equilibrium point zero. From Theorem 2, we get the following conclusion.

Corollary 1 Assume that $\left(A_{1}\right),\left(A_{2}\right)$ with $\hat{J}=0$ hold. Then the zero solution of $(1)$ is globally exponentially stable in Lyapunov sense and the exponential convergence rate is determined by (21).

Theorem 3 Assume that $\left(A_{1}\right),\left(A_{2}\right)$ hold. Then $S=\left\{\phi \in C \mid[\phi]_{\tau}^{+} \leq(E-|C|)^{-1} \hat{\Pi}^{-1} \hat{J},\left[\phi\left(t_{0}\right)-\right.\right.$ $\left.\left.C \phi\left(-r\left(t_{0}\right)\right)\right]^{+}=\left[(E-|C|)[\phi]_{\tau}^{+}\right]^{+}\right\}$is a positive invariant set and also a global attracting set of (1).

Proof Since $[\phi]_{\tau}^{+} \leq(E-|C|)^{-1} \hat{\Pi}^{-1} \hat{J}$ and $\left[\phi\left(t_{0}\right)-C \phi\left(-r\left(t_{0}\right)\right)\right]^{+}=\left[(E-|C|)[\phi]_{\tau}^{+}\right]^{+}$, then from the definition of $u(t)$ and $\omega(t)$, we get

$$
u(t) \leq \hat{\Pi}^{-1} \hat{J} \quad \text { and } \quad \omega(t) \leq(E-|C|)^{-1} \hat{\Pi}^{-1} \hat{J}, \quad t_{0}-\tau \leq t \leq t_{0}
$$

We choose $k=0$ in Theorem 1; the remaining proof is similar to the proof of Theorem 2, and we omit it here. So we get the conclusion.

If we further assume that $c_{i j}=0, i, j \in \mathcal{N}$, then the system (1) becomes

$$
\left\{\begin{array}{l}
x_{i}^{\prime}(t)=-d_{i} x_{i}(t)+\sum_{j=1}^{n} a_{i j} f_{j}\left(x_{j}(t)\right)+\sum_{j=1}^{n} b_{i j} g_{j}\left(x_{j}\left(t-\tau_{i j}(t)\right)\right)+J_{i}, \quad t \geq t_{0}, \\
x_{i}\left(t_{0}+s\right)=\phi_{i}(s), \quad-\tau \leq s \leq 0, i \in \mathcal{N} .
\end{array}\right.
$$


Therefore, we can get the following corollary.

Corollary 2 Assume that $\left(A_{1}\right)$ and $\left(A_{2}\right)$ with $c_{i j}=0, i, j \in \mathcal{N}$ hold. Then $S=\left\{\phi \in C \mid[\phi]_{\tau}^{+} \leq\right.$ $\left.(D-\hat{A}-\hat{B})^{-1} \hat{J}\right\}$ is a positive invariant set and also a global attracting set of (26).

Remark 2 The authors in [23] consider the special case of the model (26), but they require that the activation functions are continuous and monotonically nondecreasing, and the delay functions are satisfying $\frac{d \tau_{i j}(t)}{d t} \leq 0$.

\section{Examples}

Example 1 Consider the nonlinear neutral differential equation with delays

$$
\left\{\begin{aligned}
x_{1}^{\prime}(t)= & -5 x_{1}(t)+g_{1}\left(x_{1}\left(t-\tau_{11}(t)\right)\right)-\frac{3}{4} g_{2}\left(x_{2}\left(t-\tau_{12}(t)\right)\right) \\
& +\frac{1}{4}\left(1+\frac{4}{3} \cos 4 t\right) x_{1}^{\prime}(t-r(t))+J_{1} \\
x_{2}^{\prime}(t)= & -4 x_{2}(t)-\frac{1}{2} g_{1}\left(x_{1}\left(t-\tau_{21}(t)\right)\right)+g_{2}\left(x_{2}\left(t-\tau_{22}(t)\right)\right) \\
& +\frac{1}{4}\left(1+\frac{4}{3} \cos 4 t\right) x_{2}^{\prime}(t-r(t))+J_{2}, \quad t \geq 0
\end{aligned}\right.
$$

where $g_{1}(s)=\frac{|s+1|-|s-1|}{2}, g_{2}(s)=s, 0<r(t)=\frac{1}{2}-\frac{1}{3} \sin 4 t \leq \frac{5}{6}<1 \triangleq \tau, \tau_{i j}(t)=|\sin (i+j) t| \leq 1 \triangleq \tau$ for $i, j=1,2$.

By simple computation, we get

$$
\begin{aligned}
& D=\left(\begin{array}{ll}
5 & 0 \\
0 & 4
\end{array}\right), \quad \hat{A}=\left(\begin{array}{ll}
0 & 0 \\
0 & 0
\end{array}\right), \\
& \hat{B}=\left(\begin{array}{ll}
1 & \frac{3}{4} \\
\frac{1}{2} & 1
\end{array}\right), \quad|C|=\left(\begin{array}{ll}
\frac{1}{4} & 0 \\
0 & \frac{1}{4}
\end{array}\right), \\
& \hat{\Pi}=-\left(-2 D+(D+\hat{A}+\hat{B})(E-|C|)^{-1}\right)=\left(\begin{array}{cc}
2 & -1 \\
-\frac{2}{3} & \frac{4}{3}
\end{array}\right), \\
& (E-|C|)^{-1} \hat{\Pi}^{-1}=\left(\begin{array}{cc}
\frac{8}{9} & \frac{2}{3} \\
\frac{4}{9} & \frac{4}{3}
\end{array}\right) .
\end{aligned}
$$

We can easily observe that $\rho(|C|)=\frac{1}{4}<1, \hat{\Pi}$ is a nonsingular $\mathcal{M}$-matrix and

$$
\Omega_{M}(\hat{\Pi})=\left\{\left(z_{1}, z_{2}\right)^{T}>0 \mid \frac{1}{2} z_{2}<z_{1}<2 z_{2}\right\} .
$$

Let $z=(1,1)^{T} \in \Omega_{M}(\hat{\Pi})$, and $\lambda=0.11$, which satisfies the inequalities

$$
\begin{aligned}
& \rho\left(e^{\lambda \tau}|C|\right)=0.2791<1, \\
& \left(\lambda E-2 D+\left(D+\hat{A}+\hat{B} e^{\lambda \tau}\right)\left(E-|C| e^{\lambda \tau}\right)^{-1}\right) z=(-0.2448,-0.0190)^{T}<\mathbf{0} .
\end{aligned}
$$

Case 1 Let $J_{1}=2, J_{2}=-2$, so by Theorem 2, we know $S=\left\{\phi \in C \mid[\phi]_{\tau}^{+} \leq(E-|C|)^{-1} \hat{\Pi}^{-1} \hat{J}=\right.$ $\left.\left(\frac{28}{9}, \frac{32}{9}\right)^{T}\right\}$ is a global attracting set of $(27)$, and by Theorem 3 , we know $S^{*}=\left\{\phi \in C \mid[\phi]_{\tau}^{+} \leq\right.$ 


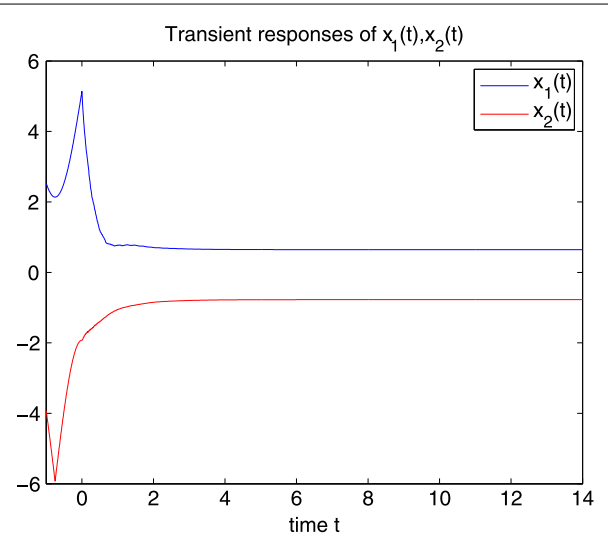

Figure 1 The state trajectory of $x_{1}(t), x_{2}(t)$ of the model (27) with $J_{1}=2, J_{2}=-2$ and initial conditions $\phi_{1}(s)=5+3 \sin \frac{2}{3} \pi s, \phi_{2}(s)=-6+4\left|\cos \frac{2}{3} \pi s\right|, s \in[-1,0]$.

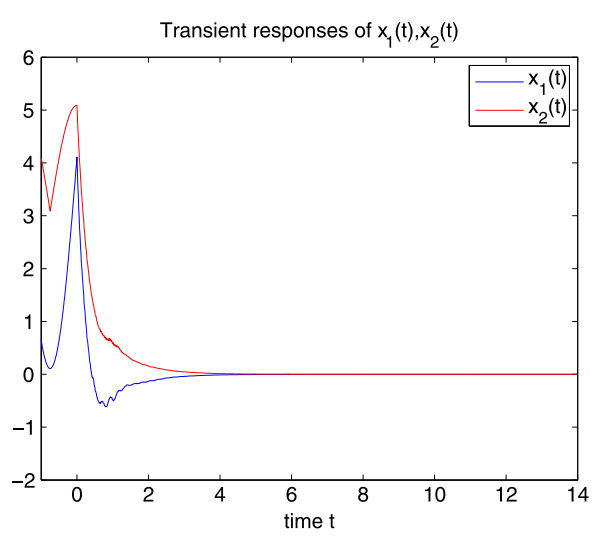

Figure 2 The state trajectory of $x_{1}(t), x_{2}(t)$ of the model (27) with $J_{1}=0, J_{2}=0$ and initial conditions $\phi_{1}(s)=4+4 \sin \frac{2}{3} \pi s, \phi_{2}(s)=3+2\left|\cos \frac{2}{3} \pi s\right|, s \in[-1,0]$.

$\left(\frac{28}{9}, \frac{32}{9}\right)^{T}$ and $\left.\phi(0)=\phi\left(-\frac{1}{2}\right)= \pm[\phi]_{\tau}^{+}\right\}$is a positive invariant and global attracting set of $(27)$. (See Figure 1.)

Remark 3 The authors in [25] considered the global attracting set of neutral type system, but the boundedness of activation functions is required, so the Theorem 1 in [25] is ineffective for the model (27).

Case 2 If $J_{1}=J_{2}=0$, from Corollary 1, we know the zero solution of (27) is globally exponentially stable in Lyapunov sense and the exponential convergence rate is equal to 0.11. (See Figure 2.)

Remark 4 It is evident that the delay functions $r(t)=\frac{1}{2}-\frac{1}{3} \sin 4 t, \tau_{i j}(t)=|\sin (i+j) t|$ do not satisfy the condition $\sup _{t \in R} \dot{r}(t)<1, \sup _{t \in R} \dot{\tau}_{i j}(t)<1, i, j=1,2$, so the results in [14, 35] are invalid for the model (27). 


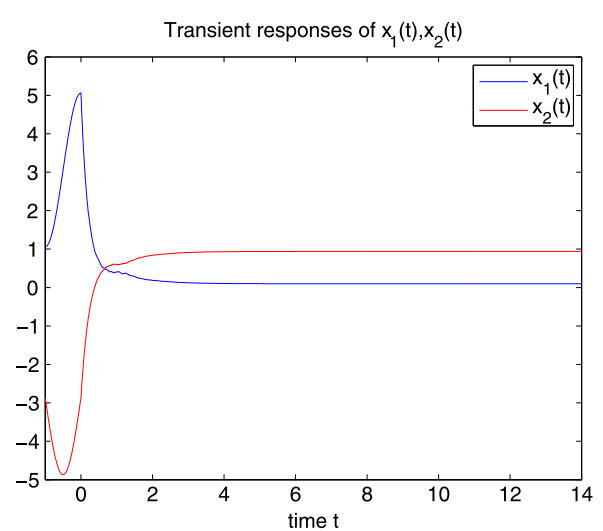

Figure 3 The state trajectory of $x_{1}(t), x_{2}(t)$ of the model (28) with initial conditions $\phi_{1}(s)=3+2 \cos \pi s$, $\phi_{2}(s)=-3+2 \sin \pi s, s \in[-1,0]$.

Example 2 Consider the nonlinear differential equation with delays

$$
\left\{\begin{array}{l}
x_{1}^{\prime}(t)=-5 x_{1}(t)+g_{1}\left(x_{1}\left(t-\tau_{11}(t)\right)\right)-\frac{3}{4} g_{2}\left(x_{2}\left(t-\tau_{12}(t)\right)\right)+1, \\
x_{2}^{\prime}(t)=-4 x_{2}(t)-\frac{1}{2} g_{1}\left(x_{1}\left(t-\tau_{21}(t)\right)\right)+g_{2}\left(x_{2}\left(t-\tau_{22}(t)\right)\right)+3, \quad t \geq 0,
\end{array}\right.
$$

where $g_{1}(s)=\frac{|s+1|-|s-1|}{2}, g_{2}(s)=\sin s, \tau_{i j}(t)=|\sin (i+j) t| \leq 1 \triangleq \tau$ for $i, j=1,2$.

Similarly to the computation of Example 1, from Corollary 2, we can get the set $S=\{\phi \in$ $\left.C \mid[\phi]_{\tau}^{+} \leq(D-\hat{A}-\hat{B})^{-1} \hat{J}=\left(\frac{14}{31}, \frac{100}{93}\right)^{T}\right\}$ is an invariant and global attracting set of the model (28). (See Figure 3.)

Remark 5 It is evident that the activation function $g_{2}(s)=\sin s$ is not monotonically nondecreasing and the delay functions $\tau_{i j}(t)=|\sin (i+j) t|$ do not satisfy $\frac{d \tau_{i j}(t)}{d t} \leq 0, i, j=1,2$, so the results in [23] are invalid for the model (28).

\section{Competing interests}

The author declares that they have no competing interests.

\section{Author's contributions}

$S J L$ carried out the main proof of the theorems and examples in this paper alone. The author approved the final manuscript.

\section{Acknowledgements}

The author sincerely thanks the editor and the reviewers for the detailed comments and valuable suggestions to improve the quality of this paper. The author would like to thank the professor Daoyi Xu of Sichuan University for his help in completing this paper. This work is supported by National Natural Science Foundation of China under Grant 10971147, Scientific Research Fund of Sichuan Provincial Education Department under Grant 10ZA032, Mathematics Tianyuan Fund under Grant 11126229 and Fundamental Research Funds for the Central Universities under Grant 2011 SCU11111.

Received: 13 January 2012 Accepted: 2 July 2012 Published: 20 July 2012

\section{References}

1. Gao, S, Chen, L, Nieto, JJ, Torres, A: Analysis of a delayed epidemic model with pulse vaccination and saturation incidence. Vaccine 24,6037-6045 (2006)

2. Mohamad, S, Gopalsamy, K: Exponential stability of continuous-time and discrete-time cellular neural networks with delays. Appl. Math. Comput. 135, 17-38 (2003)

3. Song, QK, Cao, JD: Stability analysis of Cohen-Grossberg neural network with both time-varying and continuously distributed delays. J. Comput. Appl. Math. 197, 188-203 (2006) 
4. Zhang, $\mathrm{Q}, \mathrm{Wei}, \mathrm{XP}, \mathrm{Xu}, \mathrm{J}$ : Delay-dependent exponential stability of cellular neural networks with time-varying delays. Chaos Solitons Fractals 23, 1363-1369 (2005)

5. Zhou, DM, Zhang, LM, CaO, JD: On global exponential stability of cellular neural networks with Lipschitz-continuous activation function and variable delays. Appl. Math. Comput. 2, 379-392 (2004)

6. Gu, HB, Jiang, HJ, Teng, ZD: Existence and globally exponential stability of periodic solution of BAM neural networks with impulses and recent-history distributed delays. Neurocomputing 71, 813-822 (2008)

7. Hale, JK, Verduyn Lunel, SM: Introduction to Functional Differential Equations. Springer, New York (1993)

8. Kolmanovskii, V, Myshkis, A: Introduction to the Theory and Applications of Functional Differential Equations. Kluwer Academic, London (1999)

9. Long, SJ, Xu, DY: Delay-dependent stability analysis for impulsive neural networks with time varying delays. Neurocomputing 71, 1705-1713 (2008)

10. Long, SJ, Xu, DY, Zhu, W: Global exponential stability of impulsive dynamical systems with distributed delays. Electron. J. Qual. Theory Differ. Equ. 10, 1-13 (2007)

11. Xu, DY, Yang, ZC: Impulsive delay differential inequality and stability of neural networks. J. Math. Anal. Appl. 305, 107-120 (2005)

12. Haddock, JR, Krisztin, T, Terjéki, J, Wu, JH: An invariance principle of Lyapunov-Razumikhin type for neutral functional differential equations. J. Differ. Equ. 107, 395-417 (1994)

13. Haddock, JR, Krisztin, T, Wu, JH: Asymptotic equivalence of neutral and infinite retarded differential equations. Nonlinear Anal. 4, 369-377 (1990)

14. Park, JH, Kwon, OM: Global stability for neural networks of neutral-type with interval time-varying delays. Chaos Solitons Fractals 41, 1174-1181 (2009)

15. Park, JH, Kwon, OM, Lee, SM: LMI optimization approach on stability for delayed neural networks of neutral-type. Appl. Math. Comput. 196, 236-244 (2008)

16. Qiu, J, Cao, J: Delay-dependent robust stability of neutral-type neural networks with time delays. J. Math. Control Sci. Appl. 1, 179-188 (2007)

17. Rakkiyappan, R, Balasubramaniam, P, Cao, JD: Global exponential stability results for neutral-type impulsive neural networks. Nonlinear Anal., Real World Appl. 11, 122-130 (2010)

18. Rojsiraphisal, T, Niamsup, P: Exponential stability of certain neutral differential equations. Appl. Math. Comput. 217, 3875-3880 (2010)

19. Wen, LP, Wang, WS, Yu, YX: Dissipativity and asymptotic stability of nonlinear neutral delay integro-differential equations. Nonlinear Anal. 72, 1746-1754 (2010)

20. Xu, DY, Yang, ZG, Yang, ZC: Exponential stability of nonlinear impulsive neutral differential equations with delays. Nonlinear Anal. 67, 1426-1439 (2007)

21. $\mathrm{Xu}, \mathrm{LG}, \mathrm{Xu}, \mathrm{DY}$ : Exponential stability of nonlinear impulsive neutral integro-differential equations. Nonlinear Anal. 69 , 2910-2923 (2008)

22. Mai, H, Liao, X, Li, C: A semi-free weighting matrices approach for neutral-type delayed neural networks. J. Comput. Appl. Math. 225, 44-55 (2009)

23. Liao, XX, Luo, Q, Zeng, ZG: Positive invariant and global exponential attractive sets of neural networks with time-varying delays. Neurocomputing 71, 513-518 (2008)

24. Tu, ZW, Jian, JG, Wang, BX: Positive invariant sets and global exponential attractive sets of a class of neural networks with unbounded time-delays. Commun. Nonlinear Sci. Numer. Simul. 16, 3738-3745 (2011)

25. Luo, Q, Zeng, ZG, Liao, XX: Global exponential stability in Lagrange sense for neutral type recurrent neural networks. Neurocomputing 74, 638-645 (2011)

26. Huang, YM, Zhu, W, Xu, DY: Invariant and attracting set of fuzzy cellular neural networks with variable delays. Appl. Math. Lett. 22, 478-483 (2009)

27. $\mathrm{Xu}, \mathrm{DY}, \mathrm{Li}, \mathrm{SY}, \mathrm{Zhou}, \mathrm{X}, \mathrm{Pu}, \mathrm{ZL}$ : Invariant set and stable region of a class of partial differential equation with time delays. Nonlinear Anal., Real World Appl. 2, 161-169 (2001)

28. Xu, DY, Yang, ZC: Attracting and invariant sets for a class of impulsive functional differential equations. J. Math. Anal. Appl. 329, 1036-1044 (2007)

29. Xu, DY, Zhao, HY: Invariant and attracting sets of Hopfield neural networks with delay. Int. J. Syst. Sci. 32, 863-866 (2001)

30. Zhao, HY: Invariant set and attractor of nonautonomous functional differential systems. J. Math. Anal. Appl. 282, 437-443 (2003)

31. Burton, TA: Differential inequalities and existence theory for differential, integral and delay equations. In: Comparison Methods and Stability Theory, pp. 35-56 (1994)

32. Liz, E, Trofimchuk, S: Existence and stability of almost periodic solutions for quasilinear delay systems and the Halanay inequality. J. Math. Anal. Appl. 248, 625-644 (2000)

33. Wang, XH, Guo, QY, Xu, DY: Exponential $p$-stability of impulsive stochastic Cohen-Grossberg neural networks with mixed delays. Math. Comput. Simul. 79, 1698-1710 (2009)

34. Wang, XH, Xu, DY: Global exponential stability of impulsive fuzzy cellular neural networks with mixed delays and reaction-diffusion terms. Chaos Solitons Fractals 42, 2713-2721 (2009)

35. Rakkiyappan, R, Balasubramaniam, P: LMI conditions for global asymptotic stability results for neutral-type neura networks with distributed time delays. Appl. Math. Comput. 204, 317-324 (2008)

36. Berman, A, Plemmons, RJ: Nonnegative Matrices in Mathematical Sciences. Academic Press, New York (1979)

37. Horn, R, Johnson, C: Matrix Analysis. Cambridge University Press, Cambridge (1985) 